\title{
Potentials Unbounded Below ${ }^{\star}$
}

\author{
Thomas CURTRIGHT
}

† CERN, CH-1211 Geneva 23, Switzerland

$\ddagger$ Department of Physics, University of Miami, Coral Gables, FL 33124-8046, USA

E-mail: curtright@physics.miami.edu

URL: http://curtright.com/

Received December 21, 2010, in final form March 27, 2011; Published online April 26, 2011

doi:10.3842/SIGMA.2011.042

\begin{abstract}
Continuous interpolates are described for classical dynamical systems defined by discrete time-steps. Functional conjugation methods play a central role in obtaining the interpolations. The interpolates correspond to particle motion in an underlying potential, $V$. Typically, $V$ has no lower bound and can exhibit switchbacks wherein $V$ changes form when turning points are encountered by the particle. The Beverton-Holt and Skellam models of population dynamics, and particular cases of the logistic map are used to illustrate these features.
\end{abstract}

Key words: classical dynamical systems; functional conjugation methods; Beverton-Holt model; Skellam model

2010 Mathematics Subject Classification: 37C99; 37D45; 37E05; 37J05; 37M99; 39B22

\section{Introduction}

The main purpose of this paper is to provide a few new results obtained from the application of functional conjugation methods - Schröder theory [17] - to problems in dynamics. Specifically, the interdisciplinary connections of the Goldstone potential [11] to the population dynamics model of Beverton and Holt [1], and of qualitatively similar potentials to the mathematically more intricate population model of Skellam [18], as described below in Section 3, are original, so far as I am aware ${ }^{1}$. For an illuminating discussion of these and other population models, and an introduction to the biology literature, see [10].

Moreover, while the discrete logistic map, $x \mapsto s x(1-x)$, has been particularly well-studied, especially since the work of Feigenbaum [9], my discussion of the potentials that underlie the map is based on a new investigation using Mathematica code to construct series solutions to several hundred orders, and then to functionally continue those series. The remarkable and interesting features of the model's phase-space trajectories are again novel, and - at least for the $s=3$ case presented in Section 4 - displayed here for the first time, so far as I know. Although the potentials for the logistic map are given by convergent series for $s \neq 1$, for $s=1$ the series for the potential is only asymptotic. Some explicit results are summarized in the Appendix for this special $s=1$ case.

\footnotetext{
*This paper is a contribution to the Proceedings of the Workshop "Supersymmetric Quantum Mechanics and Spectral Design" (July 18-30, 2010, Benasque, Spain). The full collection is available at http://www.emis.de/journals/SIGMA/SUSYQM2010.html

${ }^{1}$ This statement is reinforced by the fact that my colleagues in mathematical ecology and biology were unaware of any such connections.
} 


\section{Formalism}

Since the functional approach is unconventional and not part of the standard syllabus for classical mechanics, to make the discussion self-contained and accessible to the reader, it is necessary to briefly review some earlier work on this subject, especially that previously presented in $[3,4,5,6]$. Of course, the number of known results in the theory of functional methods is considerable, and the mathematical literature on the subject is vast. For a thorough survey of those results and an excellent guide to that literature, see [13].

\subsection{The problem}

Given a discrete map (unit time step, discrete scale change, etc.),

$$
x \mapsto f_{1}(x),
$$

how do we interpolate (for intermediate times, scales, etc.)? Such an interpolation is needed to interpret this dynamical system as a continuous flow (Hamiltonian, renormalization, etc.) sampled discretely.

As we have emphasized in recent papers [4, 5, 3, 6], there is an elegant method, based on the work of Ernst Schröder [17], that exploits analyticity in $x$ to find an interpolation in $t$.

\subsection{The method}

Schröder's functional equation is

$$
s \Psi(x, s)=\Psi\left(f_{1}(x, s), s\right),
$$

for some parameter (eigenvalue) $s$, with $\Psi$ to be determined. So $f_{1}$ is the functional conjugation of the eigen-scale-parameter $s$,

$$
f_{1}(x, s)=\Psi^{-1}(s \Psi(x, s), s),
$$

where the inverse function $\Psi^{-1}$ obeys Poincaré's equation [16],

$$
\Psi^{-1}(s x, s)=f_{1}\left(\Psi^{-1}(x, s), s\right) .
$$

The $n$th iterate of the functional equation gives

$$
s^{n} \Psi(x, s)=\Psi\left(f_{1}\left(\ldots f_{1}\left(f_{1}(x, s), s\right) \ldots, s\right), s\right),
$$

with $f_{1}$ acting $n$ times, and thus the $n$th order functional composition is also given by a functional conjugation,

$$
f_{n}(x, s) \equiv f_{1}\left(\ldots f_{1}\left(f_{1}(x, s), s\right) \ldots, s\right)=\Psi^{-1}\left(s^{n} \Psi(x, s), s\right) .
$$

In principle, with $\Psi$ in hand for a particular problem, any functional composition of $f_{1}$ can be constructed.

\subsection{The solution}

A continuous interpolation between the integer points is then given by functional conjugation, for any $t$,

$$
f_{t}(x, s)=\Psi^{-1}\left(s^{t} \Psi(x, s), s\right) .
$$

This can be a well-behaved and single-valued function of $x$ and $t$ provided that $\Psi^{-1}(x, s)$ is a well-behaved, single-valued function of $x$, even though $\Psi(x, s)$ might be, and typically is, multi-valued.

For generic real values of $\Psi$ appearing in the argument of $\Psi^{-1}$ then: 
- When $s$ is real and $s>1$, the $t \rightarrow \infty$ behavior for $f_{t}(x, s)$ is determined by the large argument asymptotic behavior of $\Psi^{-1}$.

- When $s$ is real and $0<s<1$, the $t \rightarrow \infty$ behavior for $f_{t}(x, s)$ is determined by the behavior of $\Psi^{-1}$ near the origin.

- When $s$ has a phase, $f_{t}(x, s)$ will be complex for generic $t$.

However, regarding this last point, although it may well have been of some interest to participants at the workshop on Supersymmetric Quantum Mechanics and Spectral Design, here I will consider only real $s>0$.

\subsection{An interpretation}

The interpolation can be envisioned as the trajectory of a particle, evolving from initial $x \equiv$ $\left.x(t)\right|_{t=0}$. Thus

$$
x(t)=f_{t}(x, s),
$$

where the particle is moving under the influence of a potential according to Hamiltonian dynamics. The velocity of the particle is then found by differentiating with respect to $t$,

$$
\frac{d x(t)}{d t}=(\ln s) s^{t} \Psi(x)\left(\Psi^{-1}\left(s^{t} \Psi(x)\right)\right)^{\prime},
$$

where any dependence of $\Psi$ on $s$ is implicitly understood. Therefore, the velocity will inherit and exhibit any multi-valued-ness possessed by $\Psi(x)$.

\subsection{The potential}

Up to an additive constant, in suitable mass units, $V(x)$ may be defined by $[4,5]$,

$$
V(x) \equiv-v^{2}(x)
$$

and its $x$ dependence follows from that of the velocity profile of the interpolation,

$$
\left.v(x) \equiv \frac{d f_{t}(x)}{d t}\right|_{t=0}=\frac{\ln s}{\frac{d}{d x} \ln \Psi(x)} .
$$

The potential $V \equiv-v^{2}$ then emerges as a quadratic in $\Psi / \Psi^{\prime}$,

$$
V(x)=-(\ln s)^{2}\left(\frac{\Psi(x)}{\Psi^{\prime}(x)}\right)^{2},
$$

where any dependence of $V$ or $\Psi$ on $s$ is again implicit.

\subsection{A functional equation for $V$}

The potential can also be determined directly from the functional equation [3] it inherits from $\Psi$. That is,

$$
V\left(f_{1}(x, s), s\right)=\left(\frac{d}{d x} f_{1}(x, s)\right)^{2} V(x, s) .
$$

If the discrete map possesses a fixed point, we may attempt to solve this functional equation for $V$ by series in $x$ about that fixed point. 
In general, if the series can be constructed, it will of course have a finite radius of convergence. However, often the series result can then be continued to other $x$ by making use of the functional equation (a technique very familiar, e.g., for the $\Gamma$ and $\zeta$ functions) so that accurate values for the potential can often be obtained for all $x$, for any $s$. Such series and continuation techniques may also be used in many, if not all cases, to determine $\Psi$ and $\Psi^{-1}$ to arbitrary precision, in principle.

\subsection{An aside}

For the corresponding supersymmetric systems,

$$
V_{ \pm}(x) \equiv-v^{2}(x) \pm i v^{\prime}(x) .
$$

This is a complex potential, even for real $s>0$. Despite the emphasis placed on such systems at the workshop on Supersymmetric Quantum Mechanics and Spectral Design, Centro de Ciencias de Benasque Pedro Pascual, where this paper was presented, I will not discuss this any further here.

\section{$3 \quad$ Elementary examples}

\subsection{The Beverton-Holt model}

Consider the rational map,

$$
x \mapsto f_{1}(x)=\frac{2 x+1}{x+2},
$$

for $-1 \leq x \leq 1$. This is known as the classic Beverton-Holt $(\mathrm{BH})$ discrete-time population model [1], to some ${ }^{2}$, in which context the population is proportional to $1+x$. But physicists especially particle physicists - know it by another name based on the underlying potential. To see what this is, consider

$$
s \Psi(x)=\Psi\left(\frac{2 x+1}{x+2}\right), \quad \text { with } \quad s=1 / 3 .
$$

This functional equation is easily solved by series about either fixed point, $x_{*}= \pm 1$, to give series that are quickly summed ${ }^{3}$. Choosing $x_{*}=+1$ results in

$$
\begin{aligned}
& \Psi(x)=\frac{2 x-2}{x+1}, \quad \Psi^{-1}(x)=\frac{2+x}{2-x}, \\
& x(t)=\Psi^{-1}\left(s^{t} \Psi(x, s), s\right)=\frac{(x+1)+(x-1)(1 / 3)^{t}}{(x+1)-(x-1)(1 / 3)^{t}}, \\
& V(x)=-v^{2}(x)=-\frac{1}{4}(\ln 3)^{2}\left(1-x^{2}\right)^{2} .
\end{aligned}
$$

We immediately recognize $V(x)$ as the Goldstone potential $[11,14]^{4}$, albeit inverted as encountered in instanton problems [15], as shown in Fig. 1.

While $\Psi$ and $\Psi^{-1}$ as given above have poles, the underlying potential in this case is an entire function, so it is a trivial step to consider particle motion not just on the original interval, but

\footnotetext{
${ }^{2}$ The model was also discussed in an earlier paper on population dynamics: $\S 4.3$ of [18] - the necessary change of variables being most readily apparent upon comparing equation (42) in [18] with (3.4) to follow.

${ }^{3}$ Schröder's equation, in particular (3.2), is homogeneous in $\Psi$. So one is free to multiply $\Psi$ by a constant. This has no effect on $v(x)$ and $V(x)$, as evident in (2.1) and (2.2), nor does it change the trajectory, so the underlying physics is the same.

${ }^{4}$ The trajectory (3.4) is the familiar "S-curve" of logistic growth, wikipedia.org/wiki/Logistic_function.
} 


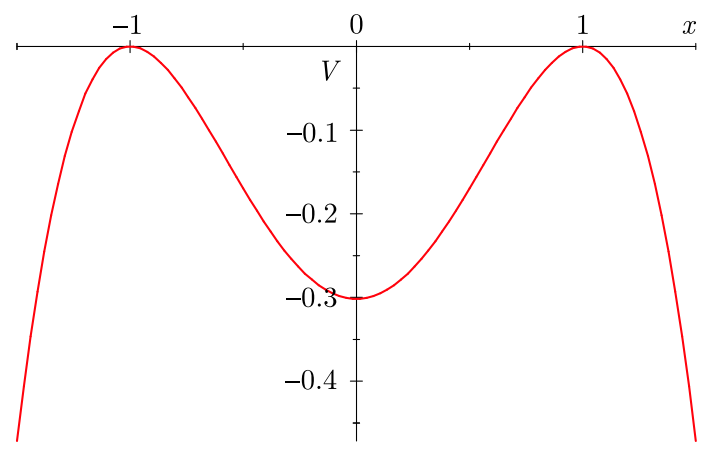

Figure 1. An inverted Goldstone potential.

on the complete real line, where $V$ is unbounded below. (This is one reason for my chosen title. Another will arise in the discussion of the logistic map in the next section.)

To be thorough, we note that Schröder's method of analysis also works for an inverted quartic potential with only one real extremum. However, in this case the relevant discrete map and continuous flow would also have purely imaginary fixed points. To understand this, consider $v(x)=1+x^{2}$ with corresponding potential

$$
V(x)=-\left(1+x^{2}\right)^{2} .
$$

Construction of the zero-energy trajectory, and a $\Psi$ function and it's inverse, follow immediately for the system governed by (3.6). Thus

$$
\begin{aligned}
& \Psi(x)=\exp (\arctan x), \quad \Psi^{-1}(x)=\tan (\ln x), \\
& x(t)=\Psi^{-1}\left(e^{t} \Psi(x)\right)=\tan (t+\arctan x) .
\end{aligned}
$$

Similarities to the $\mathrm{BH}$ case are highlighted by the discrete time-step map, now given by

$$
x \mapsto f_{\pi / 4}(x)=\tan \left(\frac{\pi}{4}+\arctan x\right)=\frac{1+x}{1-x},
$$

to be compared to (3.1). The corresponding functional equation is

$$
e^{\pi / 4} \Psi(x)=\Psi\left(\frac{1+x}{1-x}\right)
$$

and is easily checked to be satisfied by $\Psi$ in (3.7) by use of the addition formula for the tangent.

It is interesting ${ }^{5}$ that the discrete map in (3.9) has period four: $f_{\pi / 4} \circ f_{\pi / 4} \circ f_{\pi / 4} \circ f_{\pi / 4}=\mathrm{id}$, i.e. the identity map, so that $f_{\pi / 4}\left(f_{\pi / 4}\left(f_{\pi / 4}\left(f_{\pi / 4}(x)\right)\right)\right)=x$. Naively, this would seem to require that the eigenvalue in Schröder's equation must be a fourth root of unity, thus either \pm 1 or $\pm i$, and not $e^{\pi / 4}$ as given in (3.10). This warrants some explanation.

The solution $\Psi(x)=\exp (\arctan x)$ was obtained by solving Schröder's equation for continuous time, namely,

$$
e^{t} \Psi(x)=\Psi(x(t))
$$

where $\left.x \equiv x(t)\right|_{t=0}$ and where the time-dependent particle trajectory to be used in (3.11) is the elementary, right-moving, zero-energy solution (3.8) for the inverted quartic potential (3.6). However, this potential deepens so rapidly that a particle moving according to (3.8) always

\footnotetext{
${ }^{5}$ I thank the anonymous referee for raising this issue.
} 
reaches $x=+\infty$ in a finite time starting from any initial $x>0$, namely, $t_{x \Rightarrow \infty}=\frac{\pi}{2}-\arctan x$. Of course, this time depends on the initial position but it always satisfies $t_{x \Rightarrow \infty}<\frac{\pi}{2}$ for any initial position satisfying $0<x<\infty$.

Now, at this juncture you could adopt the narrow point of view that it makes no physical sense to consider any solution for larger times $t>t_{x \Rightarrow \infty}$. This would preclude taking $t=\pi$ as required to produce $f_{\pi / 4} \circ f_{\pi / 4} \circ f_{\pi / 4} \circ f_{\pi / 4}=f_{\pi}=\mathrm{id}$. And that would be the end of our story.

On the other hand, if you were just a bit more flexible in your thinking, you might consider the particle to be moving on a circle, albeit of infinite radius, and identify the point $x=+\infty$ with the point $x=-\infty$. Then for time $t>t_{x \Rightarrow \infty}$ the particle's zero-energy motion would continue, except that the particle would be coming in from $x=-\infty$ and approaching $x=0$ from the left. But, for zero total energy in the inverted quartic potential (3.6), it would again take the particle only a finite time to go from $x=-\infty$ to $x=0$, namely, $t_{-\infty \Rightarrow 0}=\frac{\pi}{2}$, and then the particle would continue moving to the right so that it would reach its original, initial position in an additional time $t_{0 \Rightarrow x}=\arctan x$.

In this second point of view, for the particle to carry out one circumnavigation of the infinite circle and return to its original position, the required total time $t_{\circlearrowright}$ is finite, due to the presence of the negative, unbounded potential. That is to say,

$$
t_{\circlearrowright}=t_{x \Rightarrow \infty}+t_{-\infty \Rightarrow 0}+t_{0 \Rightarrow x}=\pi .
$$

This is independent of the initial $x$, and it corresponds precisely to the statement that $f_{\pi / 4} \circ$ $f_{\pi / 4} \circ f_{\pi / 4} \circ f_{\pi / 4}(x)=f_{\pi}(x)=x$, the identity map.

Moreover, since the particle's instantaneous transition from $x=+\infty$ to $x=-\infty$ is, mathematically, just a change from one branch of the arctan function to the next, for the solution under consideration, Schröder's equation is indeed satisfied when $t=\pi$ but only in the following sense:

$$
e^{\pi} \Psi_{n \text {th } \operatorname{branch}}(x)=\Psi_{n+1 \text { st } \operatorname{branch}}(x),
$$

where $\Psi_{n \text {th branch }}(x)=\exp \left(\arctan _{n \text {th branch }}(x)\right)=\exp \left(n \pi+\arctan _{\text {principal branch }}(x)\right)$.

Perhaps a better way to describe all this is to project a circle of unit diameter onto a tangent line. Then a zero-energy trajectory along the $x$-axis defined by that tangent, in an inverted quartic potential as described above, corresponds precisely to constant angular velocity $(\omega=1)$ around the circle. This is the entire content of (3.8). In that expression the argument $\theta=$ $t+\arctan x$ is just the angle between the circle's diameter perpendicular to the tangent and a line from the point on the circle farthest from the tangent to the point at position $x$ along the tangent line. When re-expressed as a function of that angle, the particle's energy functional becomes

$$
E[x]=(d x / d t)^{2}-\left(1+x^{2}\right)^{2} \Longrightarrow E[x(\theta)]=\left((d \theta / d t)^{2}-1\right) / \cos ^{4} \theta .
$$

To conclude this subsection, it is appropriate to point out that both the models described here are PT symmetric, i.e. invariant under $x \mapsto-x$ and $t \mapsto-t$. And indeed, in keeping with one of the prevalent themes of the workshop on Supersymmetric Quantum Mechanics and Spectral Design, the quartic model's potential (3.6) differs from the BH model's (3.5) simply by a change of time scale and the complexification: $1-x^{2} \rightarrow 1-(i x)^{2}$.

\subsection{The Skellam model}

The discrete time-stepped map in this case is

$$
x \mapsto k\left(1-e^{-x}\right),
$$


where $k$ is a real parameter. For population dynamics, only $0 \leq x<\infty$ is of interest, but the model is well-defined for all the reals (and $\mathbb{C}$ as well). Fixed points are $x=0$ and $x_{*}(k)=$ $k+$ LambertW $\left(-k e^{-k}\right)$. The nontrivial fixed point $x_{*}$ is real and positive for $k>1$, but real and negative for $0<k<1$, when the appropriate branch of the Lambert function is used. For $k=1$ there is only the trivial fixed point. This is evident from graphs of the map, for various $k$.



Figure 2. Skellam map plotted for various $k$ (namely, $1 / 2$ in red, 1 in green, $3 / 2$ in orange, and 2 in blue). Dots are the fixed points, $x_{*}(k)$.

This model of population dynamics first appeared in equation (39), §4 of [18]. (After rescaling variables: Skellam's $\Gamma H \chi=x$ here, and his $\Gamma H=k$. In fact, in his subsequent discussion of $\S 4.3$, Skellam anticipated the Beverton-Holt model by approximating the exponential in (3.12) for small $x: \exp (-x) \approx(1-x / 2) /(1+x / 2)$, the difference being $\frac{1}{12} x^{3}-\frac{1}{12} x^{4}+\frac{13}{240} x^{5}+O\left(x^{6}\right)$.) The various functional equations associated with (3.12) are:

$$
\begin{aligned}
& k \Psi(x, k)=\Psi\left(k\left(1-e^{-x}\right), k\right), \\
& \Psi^{-1}(k x, k)=k\left(1-e^{-\Psi^{-1}(x, k)}\right), \\
& k^{2} e^{-2 x} V(x, k)=V\left(k\left(1-e^{-x}\right), k\right) .
\end{aligned}
$$

It is straightforward to obtain the first few terms in a series solution of these functional equations. Unfortunately, unlike the BH case, it is not possible to express the series in closed-form as known functions, so far as I am aware. Nevertheless, the series can be used to compute the potentials numerically.

For example, the potential $V$, expanded about 0 , is:

$$
\begin{aligned}
V(x, k)= & -(\ln k)^{2}\left(x^{2}-\frac{1}{k-1} x^{3}-\frac{1}{12} \frac{k-7}{(k+1)(k-1)^{2}} x^{4}\right. \\
& \left.+\frac{1}{4\left(k+k^{2}+1\right)(k-1)^{2}} x^{5}+O\left(x^{6}\right)\right) .
\end{aligned}
$$

An alternate series solution for the potential, expanded about $x_{*}$, is:

$$
\begin{aligned}
V(x, k)= & -(\ln \ell)^{2}\left(y^{2}-\frac{1}{\ell-1} y^{3}-\frac{1}{12} \frac{\ell-7}{(\ell+1)(\ell-1)^{2}} y^{4}\right. \\
& \left.+\frac{1}{4\left(\ell+\ell^{2}+1\right)(\ell-1)^{2}} y^{5}+O\left(y^{6}\right)\right)
\end{aligned}
$$


where $y \equiv x-x_{*}$ and $\ell \equiv k-x_{*}=-$ LambertW $\left(-k e^{-k}\right)$. And yes, the coefficients in these two series do have exactly the same form.

While the functional equation for $V$ does not determine the normalization, the relative normalization for the two series (3.16) and (3.17) has been fixed by the requirement that they match on the interval $0<x<x_{*}$. With the relative normalization as given, there is also a duality between the right-hand sides of (3.16) and (3.17) to the orders shown: $x, k \Longleftrightarrow y, \ell$. Under this interchange the expressions are identical in form, as already noted. This equivalence between the two series expansions can be established to all orders by inspection of the corresponding recursion relations for the series coefficients.

For all positive $k>1$ the potentials for the Skellam model are qualitatively similar to the inverted Goldstone potential, just not as symmetrical. Outside the regions between the two real fixed points, which are local maxima, the potentials are apparently unbounded below. Between the fixed points the potentials have a single local minimum at $x_{\min }(k) \approx x_{*} / 2$. For any $k>1$, the leading $x^{2}$ and $y^{2}$ behavior of $V$ in (3.16) and (3.17) show that an infinite amount of time is required for a zero-energy trajectory to reach the lower and upper fixed points, respectively, starting from somewhere else. Consequently, the Schröder function constructed about $x=0$, with $\Psi(x)=x+O\left(x^{2}\right)$, will truly diverge to $+\infty$ as $x \rightarrow x_{*}$. Another way to say this is that $\Psi^{-1}$ will asymptote to a constant as its argument becomes large, $\lim _{z \rightarrow \infty} \Psi^{-1}(z, k)=x_{*}$, when $k>1$.

For positive $k<1$, the Skellam model potentials also have structure qualitatively similar to the inverted Goldstone potential, except that the nontrivial local maximum and the local minimum are at negative $x$. For $k=1$ the potential is best defined as a limit, $V(x, 1)=$ $\lim _{k \rightarrow 1} V(x, k)=-\frac{1}{4} x^{4}-\frac{1}{12} x^{5}+O\left(x^{6}\right)$. However, in this latter case the series for $V(x, 1)$ is probably not convergent, but asymptotic instead. (We discuss in some detail a similar situation for a special case of the logistic map, in the Appendix. The corresponding analysis of the $k=1$ Skellam model is left as an exercise for the reader.)

We plot the potentials in the region between the two fixed points for a few representative values of $k>1$, using the series expansions (3.16) and (3.17), only extended to tenth order in $x$ and $y$. This order is sufficient for any errors in the graphs to be insignificant, and indiscernible.

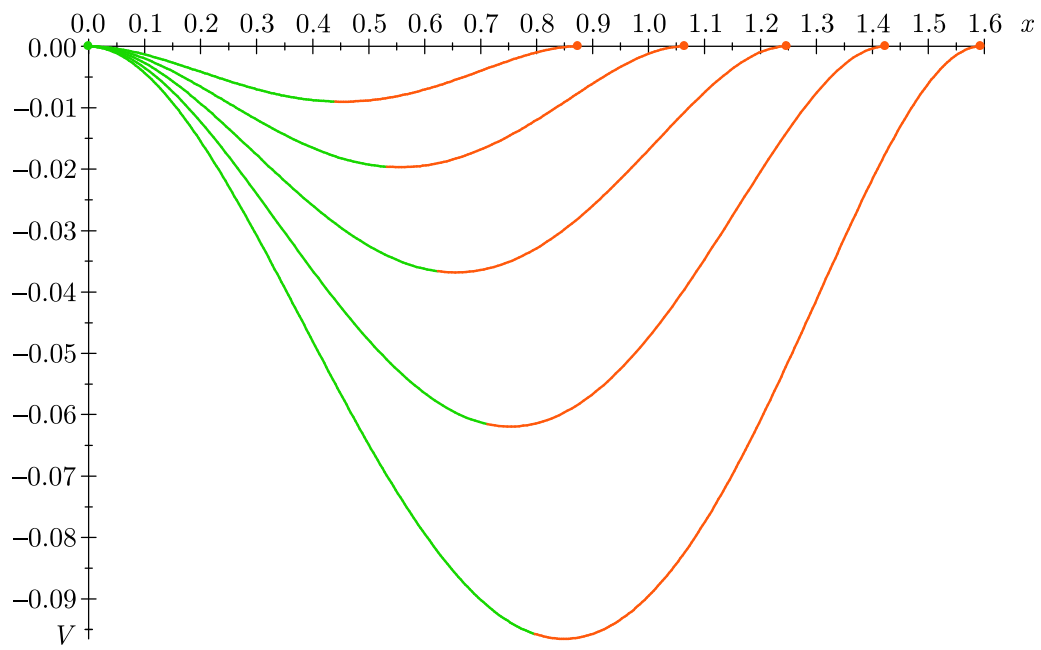

Figure 3. Skellam model potentials, $V(x, k)$, for $k=3 / 2,13 / 8,7 / 4,15 / 8$, and 2 (as successively lower curves) from the series about 0 (in green) joined at $x_{*} / 2$ to the series about $x_{*}$ (in orange). Dots on the $x$ axis are the fixed points.

The continuous trajectories for the Skellam model can also be computed numerically, although they are not known in simple, closed form. The most direct way to do this is to compute the 
inverse of the Schröder function from (3.14), say as series, and then re-use that functional equation to improve upon the numerical results obtained from the series. Since this article is mainly about the potentials that underlie the trajectories, I will not present here numerical results for $\Psi^{-1}$. But I will discuss the series solution of (3.14) for generic $k$.

The functional equation for $\Psi^{-1}$ is interesting in its own right. Let $\Psi^{-1}(x, k)=-f(-x, k)$, then (3.14) becomes

$$
f(k x, k)=k\left(e^{f(x, k)}-1\right) .
$$

Consider a formal series solution:

$$
f(x, k)=\sum_{j=1}^{\infty} \frac{b_{j} x^{j}}{j !} .
$$

The exponential formula ${ }^{6}$,

$$
\exp \left(\sum_{j=1}^{\infty} \frac{b_{j} x^{j}}{j !}\right)=\sum_{n=0}^{\infty} \frac{B_{n}\left(b_{1}, \ldots, b_{n}\right)}{n !} x^{n}
$$

where $B_{n}\left(b_{1}, \ldots, b_{n}\right)$ are the complete multi-variate Bell polynomials ${ }^{7}$, permits us to write the functional equation (3.18) as

$$
\sum_{j=1}^{\infty} \frac{b_{j} k^{j} x^{j}}{j !}=k \sum_{n=1}^{\infty} \frac{B_{n}\left(b_{1}, \ldots, b_{n}\right)}{n !} x^{n},
$$

where it is understood that the $b_{j}$ are $k$-dependent. Thus the coefficients of $x^{n}$ give the recursion relation,

$$
k^{n-1} b_{n}=B_{n}\left(b_{1}, \ldots, b_{n}\right) .
$$

Since all of these complete Bell polynomials depend on their last argument only as $B_{n}\left(b_{1}, \ldots, b_{n}\right)$ $=b_{n}+\cdots$, with no other dependence on $b_{n}$, we can rewrite (3.20) in a technically sweeter form:

$$
b_{n}=\frac{1}{k^{n-1}-1} B_{n}\left(b_{1}, b_{2}, \ldots, b_{n-1}, 0\right) .
$$

Moreover, since the $B_{n}$ are homogeneous of degree $n$ under the multi-variate rescaling $b_{k} \rightarrow r^{k} b_{k}$, without loss of generality we may choose $b_{1}=1$, thereby setting the scale of $x$ in the expansion $(3.19)^{8}$.

Because of the prefactor in (3.21), we see that each $b_{n+1}$ is a polynomial in $k$ multiplied by a factor $1 / \prod_{m=1}^{n}\left(k^{m}-1\right)$, and thus singular as $k \rightarrow 1$. More precisely, I find $b_{n+1}(k) \underset{k \rightarrow 1}{\sim}$ $\frac{1}{(k-1)^{n}}(n+1) !\left(\frac{1}{2}\right)^{n}$. So, writing

$$
b_{n+1}(k)=\frac{p_{n}(k)}{\prod_{m=1}^{n}\left(k^{m}-1\right)},
$$

\footnotetext{
${ }^{6}$ http://en.wikipedia.org/wiki/Exponential_formula.

${ }^{7}$ http://en.wikipedia.org/wiki/Bell_polynomial\#Complete_Bell_polynomials.

${ }^{8}$ From (3.21) one can show the series (3.19) converges for all $x$ when $k>1$.
} 
the resulting $p_{n}(k)$ are polynomials in $k$ of order $n(n-1) / 2$. With the choice of initial condition $b_{1}=1$, these polynomials have only positive integer coefficients. The first few are:

$$
\begin{aligned}
p_{1}(k)= & 1, \quad p_{2}(k)=2+k, \quad p_{3}(k)=6+6 k+5 k^{2}+k^{3}, \\
p_{4}(k)= & 24+36 k+46 k^{2}+40 k^{3}+24 k^{4}+9 k^{5}+k^{6}, \\
p_{5}(k)= & 120+240 k+390 k^{2}+480 k^{3}+514 k^{4}+416 k^{5}+301 k^{6}+160 k^{7}+64 k^{8}+14 k^{9}+k^{10}, \\
p_{6}(k)= & 720+1800 k+3480 k^{2}+5250 k^{3}+7028 k^{4}+8056 k^{5}+8252 k^{6}+7426 k^{7} \\
& +5979 k^{8}+4208 k^{9}+2542 k^{10}+1295 k^{11}+504 k^{12}+139 k^{13}+20 k^{14}+k^{15},
\end{aligned}
$$

etc. From these, I infer two particular values of the polynomials, for any $n$,

$$
\left.p_{n}(k)\right|_{k=0}=n !,\left.\quad p_{n}(k)\right|_{k=1}=n !(n+1) !\left(\frac{1}{2}\right)^{n} .
$$

In fact, the coefficients of the lowest two and highest two powers of $k$ are easily determined for any $n$,

$$
p_{n}(k)=n !+\frac{1}{2}(n-1) n ! k+\cdots+\frac{1}{2}(n+2)(n-1) k^{n(n-1) / 2-1}+k^{n(n-1) / 2} .
$$

This result may be established by induction, and probably extended to other coefficients as well, given enough time and interest.

In terms of the $p_{n} \mathrm{~s}$, the formal series solution for $\Psi^{-1}$ is

$$
\Psi^{-1}(x, k)=\sum_{n=1}^{\infty} \frac{(-1)^{n-1} b_{n}(k) x^{n}}{n !}=x+x \sum_{n=1}^{\infty} \frac{1}{(n+1) !} \frac{p_{n}(k) x^{n}}{\prod_{i=1}^{n}\left(1-k^{i}\right)} .
$$

We may also express this in terms of deformed, $k$-bracket factorials ${ }^{9}$,

$$
[n]_{k} !=\prod_{m=1}^{n}[m]_{k}, \quad[m]_{k}=\frac{k^{m}-1}{k-1} .
$$

This makes it clear that the series is actually in terms of the rescaled variable $x /(1-k)$. Thus,

$$
\Psi^{-1}(x, k)=x+x \sum_{n=1}^{\infty} \frac{1}{(n+1) !} \frac{p_{n}(k)\left(\frac{x}{1-k}\right)^{n}}{[n]_{k} !} .
$$

As a special case, this has a simple limit as $k \rightarrow 0$, namely,

$$
\lim _{k \rightarrow 0}\left(\Psi^{-1}(x, k)\right)=x+x \sum_{n=1}^{\infty} \frac{1}{(n+1) !} \frac{p_{n}(0) x^{n}}{1}=x+x \sum_{n=1}^{\infty} \frac{1}{n+1} x^{n}=-\ln (1-x) .
$$

Another special case yields a direct connection to the BH model, also by taking a limit. Again interchanging limit with summation we find

$$
\begin{aligned}
\lim _{k \rightarrow 1}\left(\frac{1}{1-k} \Psi^{-1}((1-k) x, k)\right) & =x+x \sum_{n=1}^{\infty} \frac{1}{(n+1) !} \frac{p_{n}(1) x^{n}}{n !} \\
& =x+x \sum_{n=1}^{\infty}\left(\frac{x}{2}\right)^{n}=\frac{2 x}{2-x}=\Psi_{\mathrm{BH}}^{-1}(x)-1,
\end{aligned}
$$

where $\Psi_{\mathrm{BH}}^{-1}$ is the inverse Schröder function in (3.3).

\footnotetext{
${ }^{9}$ http://en.wikipedia.org/wiki/Q-bracket\#Relationship_to_the_q-bracket_and_the_q-binomial.
} 
A numerical study of $\Psi^{-1}$ and the corresponding continuous trajectories for the Skellam model for general $k$ will have to be presented elsewhere. For $k \neq 1$, the trajectories are qualitatively the same as the "S-curves" of the BH model, as can be inferred just from the qualitative similarity of the inverted Goldstone potential to the Skellam model potentials, such as those exhibited in the Figure above.

Further features of the functional formalism will now be explored for a different, perhaps better-known class of models.

\section{The logistic map}

A well-studied and interesting map is given by $[2,7]$

$$
x \mapsto s x(1-x),
$$

on the unit interval, $x \in[0,1]$, for parameter values $s \in[0,4]$. The maximum of the map is $s / 4$, obtained from $x=1 / 2$, so without loss of any essential features, for a given $s$, we need only consider $x \in[0, s / 4]$. The map has fixed points at $x=0$ and at $x_{*}=1-1 / s$.

Iteration of this map shows a wealth of interesting, well-known features ${ }^{10}$.

- With $0<s<1, x_{n} \underset{n \rightarrow \infty}{\rightarrow} 0$ independent of the initial $x$.

- With $1<s \leq 2, x_{n} \underset{n \rightarrow \infty}{\rightarrow} 1-\frac{1}{s}$, monotonically.

- With $2<s \leq 3, x_{n} \underset{n \rightarrow \infty}{\rightarrow} 1-\frac{1}{s}$, oscillatorially.

- With $3<s \leq 1+\sqrt{6} \approx 3.45, x_{n}$ oscillates between two ( $s$ dependent) values.

- With $3.45 \lesssim s \lesssim 3.54, x_{n}$ oscillates between four ( $s$ dependent) values. Etc. - the so-called "period-doubling cascade".

- At $s \approx 3.57$ the period-doubling cascade ends, and the $x_{n}$ sequence becomes chaotic. Slight variations in the initial $x$ yield dramatically different results for the sequence of $x_{n}$.

- Chaotic behavior holds for almost all larger $s$ up to and including $s=4$.

Schröder's equation for the logistic map is

$$
s \Psi(x, s)=\Psi(s x(1-x), s) .
$$

The inverse function satisfies the corresponding Poincaré equation,

$$
\Psi^{-1}(s x, s)=s \Psi^{-1}(x, s)\left(1-\Psi^{-1}(x, s)\right) .
$$

As originally obtained by Schröder, there are three closed-form solutions known, for $s=-2,2$, and $4(s=-2$ is related to $s=4$ by functional conjugation):

$$
\begin{array}{lll}
\Psi(x, 2) & =-\frac{1}{2} \ln (1-2 x), & \Psi^{-1}(x, 2)=\frac{1}{2}\left(1-e^{-2 x}\right), \\
\Psi(x, 4)=(\arcsin \sqrt{x})^{2}, & \Psi^{-1}(x, 4)=(\sin \sqrt{x})^{2} .
\end{array}
$$

Note that $\Psi(x, 4)$ is multi-valued, but $\Psi^{-1}(x, 4)$ is single-valued, for $0 \leq x \leq 1$.

While the solutions for $\Psi$ for other values of $s$ are not known in a simple closed form, they may be constructed as convergent series. The functional method then reveals that there are welldefined expressions for the continuous time interpolation of the logistic map for all $s$ values.

\footnotetext{
${ }^{10}$ http://en.wikipedia.org/wiki/Logistic_map.
} 
Moreover, the potentials needed to produce the explicit, continuously evolving trajectories of the envisioned particle, for all $s$, can now be obtained. These potentials had not been obtained prior to our work, so far as we can tell from the literature. For $s>3$ these potentials are unbounded below in an interesting way.

A new feature for the potentials so obtained is that they show the "switchback effect": If and when the particle reaches a turning point, the potential changes form. This requires a bit of explanation.

\subsection{The $s=4$ case in more detail}

The trajectory, velocity, and potential are given in this case by

$$
\begin{aligned}
& x(t)=\Psi^{-1}\left(4^{t} \Psi(x, 4), 4\right)=\left(\sin \left(2^{t} \arcsin \sqrt{x}\right)\right)^{2}, \\
& \frac{d x(t)}{d t}=(\ln 4) \sqrt{x(t)(1-x(t))} \arcsin \sqrt{x(t)}, \\
& V(x(t))=(\ln 4)^{2} x(t)(x(t)-1) \arcsin ^{2} \sqrt{x(t)} .
\end{aligned}
$$

The last expression appears to be time-translationally invariant. However, the velocity function has branch points (i.e. turning points) at $x(t)=0$ and $x(t)=1$, so some care is needed to determine which branch of the potential function is actually in effect, at any given time, since the turning points are encountered at finite times.

Initially, the particle is clearly moving in a potential where all the functions are principal valued for $x>0$,

$$
V_{0}(x)=(\ln 4)^{2} x(x-1) \arcsin ^{2} \sqrt{x} .
$$



Figure 4. The primary potential, $V_{0}(x)$.

This potential is again unbounded below, as $x \rightarrow-\infty$, but the structure for $x>0$ is more interesting. So, consider the particle to be moving to the right, with initial $x>0$.

Since the RHS of $V_{0}$ does not have a reflection-free, "infinity pool" edge, the zero-energy particle initially moving to the right will reach $x=1$ in a finite time,

$$
\Delta t_{0}(x)=\frac{1}{\ln 4} \int_{x}^{1} \frac{d y}{\sqrt{y(1-y)}(\arcsin \sqrt{y})}=\frac{1}{\ln 2} \ln \left(\frac{\pi / 2}{\arcsin \sqrt{x}}\right) .
$$

It turns around at this time, but its subsequent motion is that of a zero-energy particle moving to the left in a modified potential, $V_{1}(x)$. This follows just from inspection of $v(t)$, and setting $V_{1}(x)=-v^{2}(t)$ after the encounter with the turning point. 
This modified potential does not have reflection-free edges at either side, as evident in its explicit expression,

$$
V_{1}(x)=(\ln 4)^{2} x(x-1)(-\pi+\arcsin \sqrt{x})^{2} .
$$

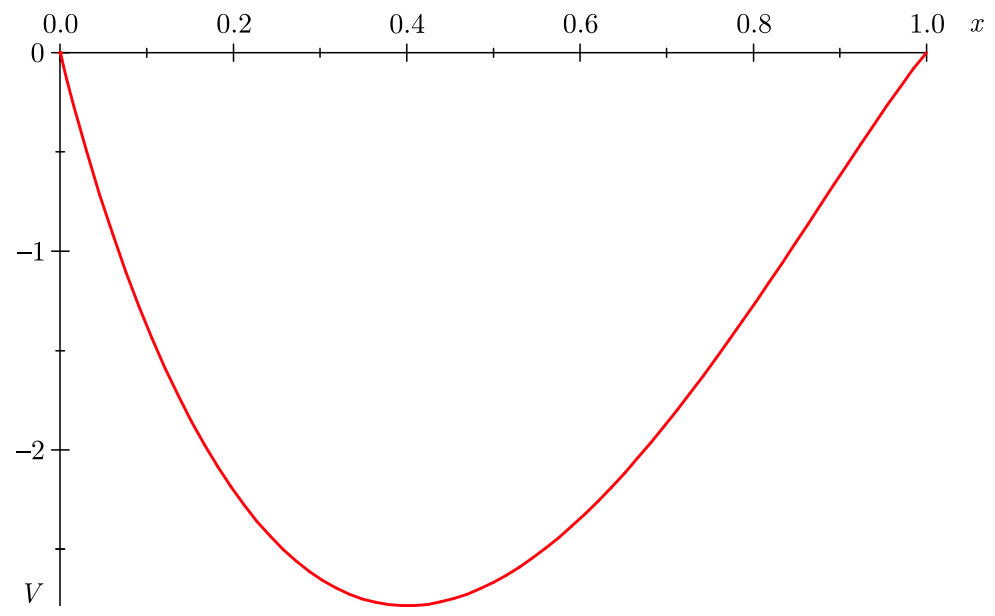

Figure 5. The first switchback potential, $V_{1}(x)$.

Moving in this modified negative potential, it now takes the zero-energy particle a finite amount of time to travel from $x=1$ down to $x=0$, as given by

$$
\Delta t_{1}=1
$$

Upon reaching $x=0$, the particle is again reflected, and the exact solution $x(t)$ exhibits another change in velocity profile that keeps $E=0$, but again alters the potential for the return trip towards $x=1$. The potential becomes

$$
V_{2}(x)=(\ln 4)^{2} x(x-1)(\pi+\arcsin \sqrt{x})^{2} .
$$

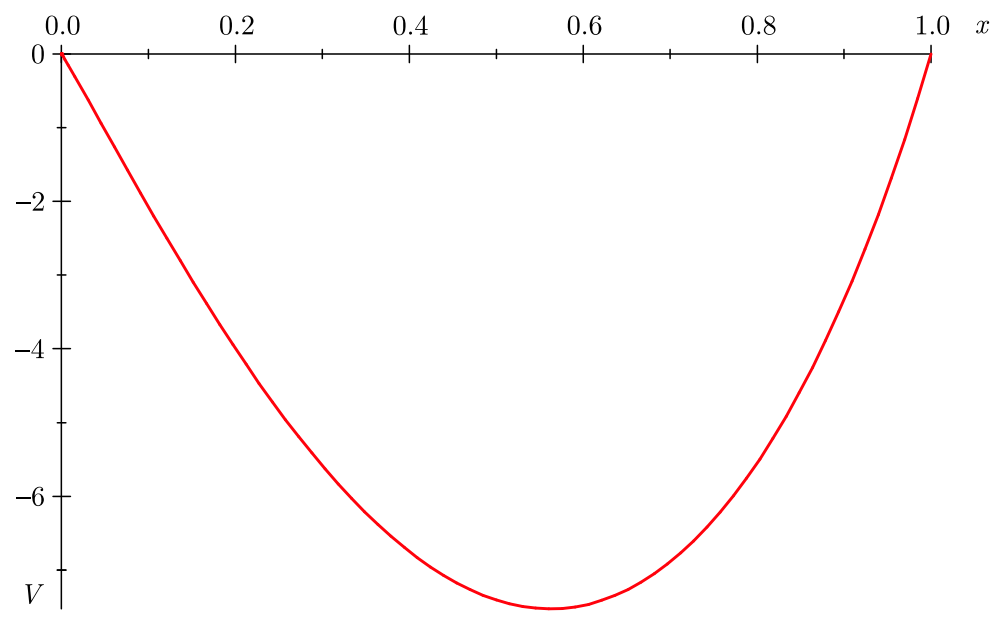

Figure 6. The second switchback potential, $V_{2}(x)$.

Notice that the sequence $V_{0} \rightarrow V_{1} \rightarrow V_{2}$ involves successively deeper, negative potentials. 
And so it goes. The motion of the particle between its encounters with the $P$ th and $(P+1)$ st turning points is determined by the potential:

$$
V_{P}(x)=(\ln 4)^{2} x(x-1)\left((-1)^{P}\left\lfloor\frac{1+P}{2}\right\rfloor \pi+\arcsin \sqrt{x}\right)^{2},
$$

where $\lfloor\cdots\rfloor$ is the floor function and arcsin is understood to take principal values. That is to say, the potential deepens as $P$ increases.

The corresponding velocity profile speeds up:

$$
v_{P}(x)=(\ln 4) \sqrt{x(1-x)}\left((-1)^{P}\left\lfloor\frac{1+P}{2}\right\rfloor \pi+\arcsin \sqrt{x}\right) .
$$

The particle will either be traveling to the left, with $v_{P}(x)<0$ for odd $P$, or traveling to the right, with $v_{P}(x)>0$ for even $P$, with its speed increasing with $P$. This effect is clearly seen upon viewing animations of the $s=4$ trajectories $^{11}$.

It is instructive to plot $E(t)=v^{2}(x(t))+V_{P}(x(t))$ versus $t$ for various $P$, to check $E=0$, as well as to see how the energy would not be conserved if the potentials were not switched.

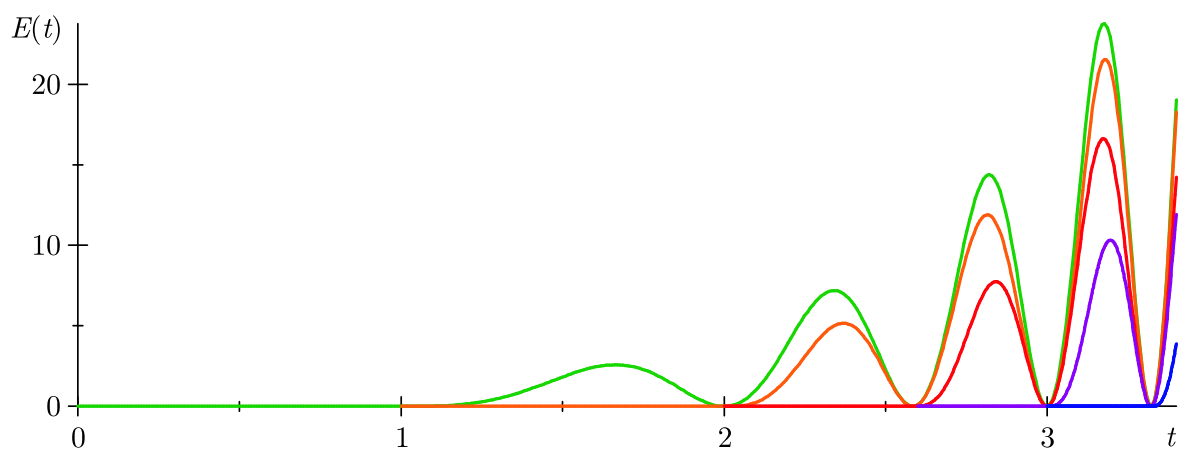

Figure 7. $E(t)$ for initial $x=1 / 2$, using potentials $V_{P}$, with $P=0,1,2,3$, and 4 , as shown in green, orange, red, purple, and blue, resp.

For particles with initial $v>0$, another way to picture the zig-zag motion of the particle is in terms of the total distance traveled. In this point of view, the successive $V_{P}$ patch together to form a continuous potential $V(X)$ on the real half-line, $X \geq 0$. Indeed, the half-line may be thought of as a covering manifold of the unit interval in $x$, with the previous multi-valued functions of $x$ now single-valued functions of $X$. In this way of looking at the particle's motion, the potential is again unbounded below (with a downward parabola as envelope), even though at any time, $x>0$.

Alternatively, the motion may be visualized as a trajectory on the sheets of a Riemann surface. Consider the particle moving on the complex $x$ plane, and not just the real line segment $[0,1]$, the endpoints of which are now branchpoints. There are cuts from +1 to $+\infty$ and from 0 to $-\infty$. The particle first moves along the real axis, approaches the cut at +1 , and then goes around the branchpoint such that

$$
\sqrt{x} \rightarrow \sqrt{x}, \quad \sqrt{1-x} \rightarrow-\sqrt{1-x}, \quad \arcsin \sqrt{x} \rightarrow \pi-\arcsin \sqrt{x} .
$$

The particle returns along the real axis to the origin and encircles the branchpoint at 0 , such that

$$
\sqrt{x} \rightarrow-\sqrt{x}, \quad \sqrt{1-x} \rightarrow \sqrt{1-x}, \quad \arcsin \sqrt{x} \rightarrow-\arcsin \sqrt{x} .
$$

\footnotetext{
${ }^{11}$ http://server.physics.miami.edu/ curtright/Schroeder.html.
} 


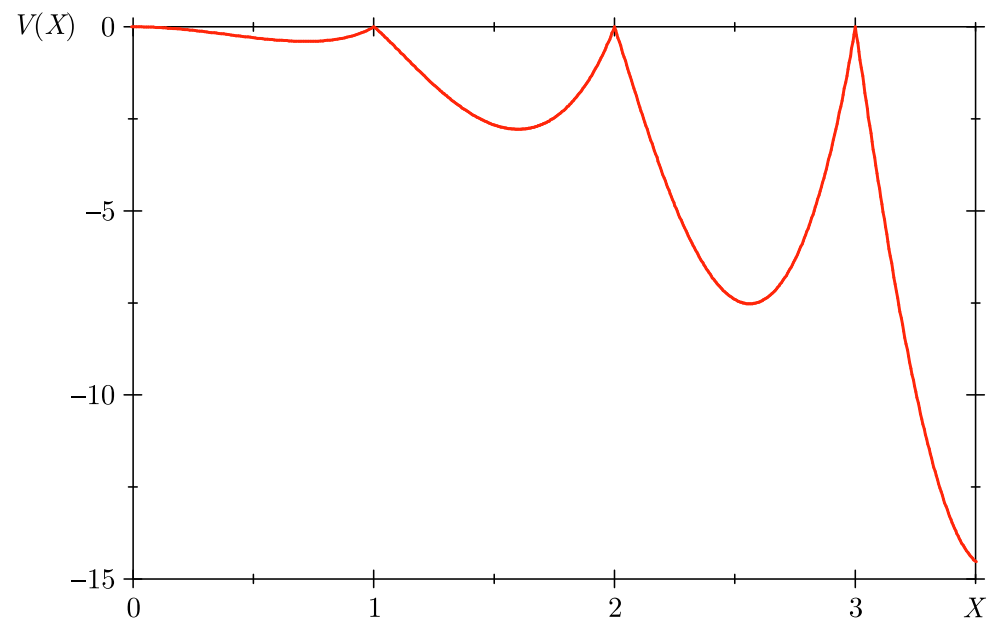

Figure 8. $V(X)$ where the total distance traveled by the particle is $X-\left.x(t)\right|_{t=0}$.

The particle then goes back to +1 and goes around it once more such that again

$$
\sqrt{x} \rightarrow \sqrt{x}, \quad \sqrt{1-x} \rightarrow-\sqrt{1-x}, \quad \arcsin \sqrt{x} \rightarrow \pi-\arcsin \sqrt{x} .
$$

The trajectory continues in this way, flipping signs and adding $\pi \mathrm{s}$ according to the formulae for $V_{P}$ and $v_{P}$.

\subsection{Series solutions for other values of $s$}

The functional equation for the potential that underlies the logistic map is

$$
V(s x(1-x), s)=s^{2}(1-2 x)^{2} V(x, s) .
$$

Applying the method of series solution about $x=0$, with initial conditions $V(0, s)=0$, $V^{\prime}(0, s)=0$, and $V^{\prime \prime}(0, s)=-2 \ln ^{2} s$, we find:

$$
\begin{aligned}
& V(x, s)=-\left(\ln ^{2} s\right) U(x, s), \\
& U(x, s)=x^{2}\left(1+\sum_{n=1}^{\infty} a_{n}(s) x^{n}\right), \quad a_{1}=\frac{2}{1-s}, \quad a_{2}=\frac{5-3 s}{(s-1)^{2}(s+1)}, \quad \ldots,
\end{aligned}
$$

provided $s \neq 1$. The higher coefficients in the expansion are determined recursively by

$$
a_{n+2}=\frac{1}{\left(1-s^{n+2}\right)}\left(4 a_{n+1}-4 a_{n}+\sum_{j=1+\left\lfloor\frac{n-1}{2}\right\rfloor}^{n+1}(-1)^{n-j} a_{j} s^{j}\left(\begin{array}{c}
j+2 \\
n+2-j
\end{array}\right)\right),
$$

for $n \geq 1$, where $\lfloor\cdots\rfloor$ is the floor function. This series solves the functional equation for any $s$, within its radius of convergence, $R(s)$. From numerical studies, that radius depends on $s$ as follows:

$$
R(s)=\frac{1}{\lim _{n \rightarrow \infty} \sup \left(\left|a_{n}(s)\right|^{1 / n}\right)}= \begin{cases}\frac{1}{2} & \text { if } 0<s \leq \frac{2}{3} \\ \left|1-\frac{1}{s}\right| & \text { if } \frac{2}{3} \leq s \leq 2 \\ \frac{s}{4} & \text { if } 2 \leq s \leq 4 .\end{cases}
$$

In particular, $R(4)=1$, as is evident from (4.1), while taking (4.3) at face value, $R(1)=0$, suggesting an asymptotic series. More on the $s=1$ case as a limit of (4.2) can be found in the Appendix. 


\subsection{Functional continuation of the series solutions}

The sequence of switchback potentials, i.e. the various branches of the analytic potential function, can be obtained from the functional equation for the potential. This follows from re-expressing the functional equation as

$$
U_{ \pm}(x)=s(s-4 x) U\left(x_{ \pm}\right), \quad x_{ \pm}=\frac{1}{2} \pm \frac{1}{2} \sqrt{1-4 x / s} .
$$

One of these potentials $\left(U_{-}\right)$reproduces the original series for the "primary potential" expanded about $x=0$, as may be seen by direct comparison of the series or by numerical evaluation. Alternatively, the other $\left(U_{+}\right)$gives the potential on another sheet of the function's Riemann surface, hence the first "switchback potential" for $s>2$. This is easily checked against the closed-form results for the $s=4$ case.

Since $U_{-}$has built into it zeroes at both $x=0$ and $x_{\max }=s / 4$, it is actually a more useful form than just the series about $x=0$. When $2<s \leq 4$, the series has radius of convergence $R(s)=s / 4$. However, from using $U_{-}$instead of the direct series, we have convergence over the whole closed interval, $x \in[0, s / 4]$, since then $0 \leq \frac{1}{2}-\frac{1}{2} \sqrt{1-4 x / s} \leq 1 / 2<R(s)$ when $2<s \leq 4$. Thus we need only evaluate $U$ appearing in $U_{-}$within the region of convergence of its series about zero. That is to say, first construct the series for $U$, and then from that series build $U_{-}$. Finally, identify this with $U_{0}$, the primary potential in the sequence.

Similarly, $U_{+}$may be identified with the first switchback in the sequence, $U_{1}$, but for better convergence properties it is useful to build $U_{+}$from $U_{0}$ instead of $U$. By doing this when

$2<s \leq 4$, we have convergence over the whole closed sub-interval, $x \in\left[\frac{1}{16} s^{2}(4-s), s / 4\right]$, with zeroes of $U_{1}$ built-in at the end-points of the interval.

This process may be continued indefinitely, through successive application of the basic substitution $U(x) \rightarrow s(s-4 x) U\left(x_{ \pm}\right)$. For example, the next set of potentials in the sequence is $U_{ \pm \pm \pm}(x)=s(s-4 x) U_{ \pm \pm}\left(x_{ \pm}\right)$, etc. In general, the $n$th iteration of the procedure gives

$$
U_{\underbrace{ \pm \pm \cdots \pm}_{n \text { times }}}(x)=s(s-4 x) U_{\underbrace{ \pm \pm \cdots \pm}_{n-1 \text { times }}}\left(x_{ \pm}\right) \text {. }
$$

Finally, at each iteration, we must select appropriate switchback potentials out of the $2^{n}$ different expressions. In particular, we note that many of the $U_{ \pm \pm \cdots \pm}(x)$ will be complex-valued for the $x$ intervals under consideration, and therefore they are not of immediate interest since they do not govern the particle's evolution along the real axis. (The continuation of the particle trajectory into the complex plane is outside the scope of this paper.)

\subsection{Numerical examples}

Consider the specific case $s=5 / 2$. This is representative for $2<s<3$. For this case, the turning points converge onto the nontrivial fixed point $x_{*}=1-1 / s=3 / 5$. This is evident in the following graphs. For $s \leq 3$, the potential sequence is actually bounded below, for positive $x$ (but not for negative $x$ ). In fact, for $s<3$ the potentials in the sequence become progressively more shallow for $x>0$ and tend to disappear!

The turning points and the behavior of the potentials near $x_{*}=3 / 5$ are shown more clearly below, for the first three potential switchbacks.

As the zero-energy particle moves through this sequence of increasingly shallow, narrowing potentials, its average speed decreases, giving the appearance of a dissipative system. Nevertheless, even as the particle motion subsides upon convergence into the fixed point at $x=3 / 5$, energy is rigorously conserved through changes in the potential.

For other values of $s$, the sequence of potentials can also be accurately determined using series solutions augmented by functional methods. 


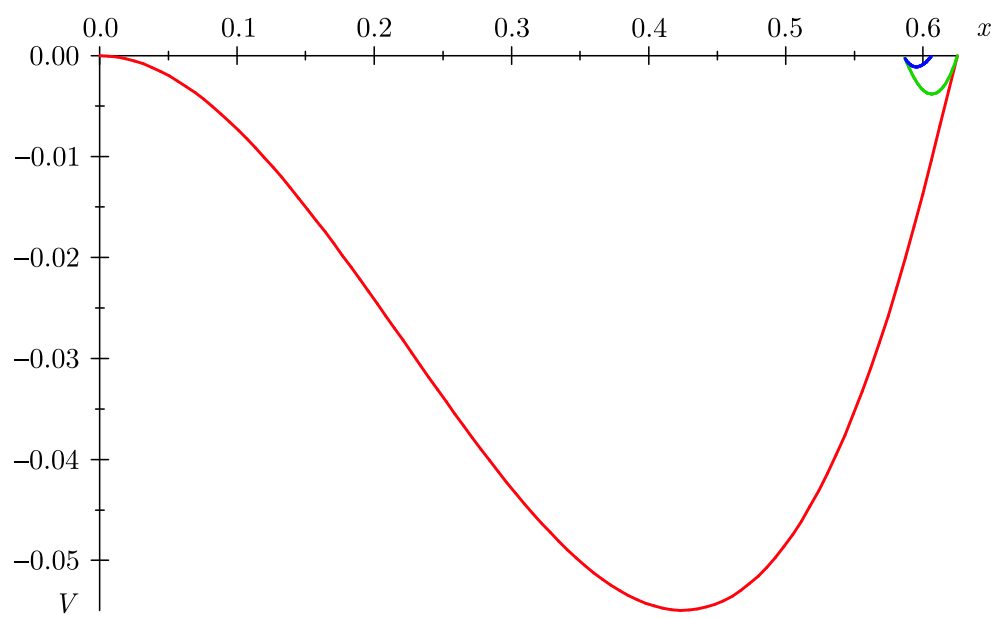

Figure 9. Logistic map potentials for $s=5 / 2$.

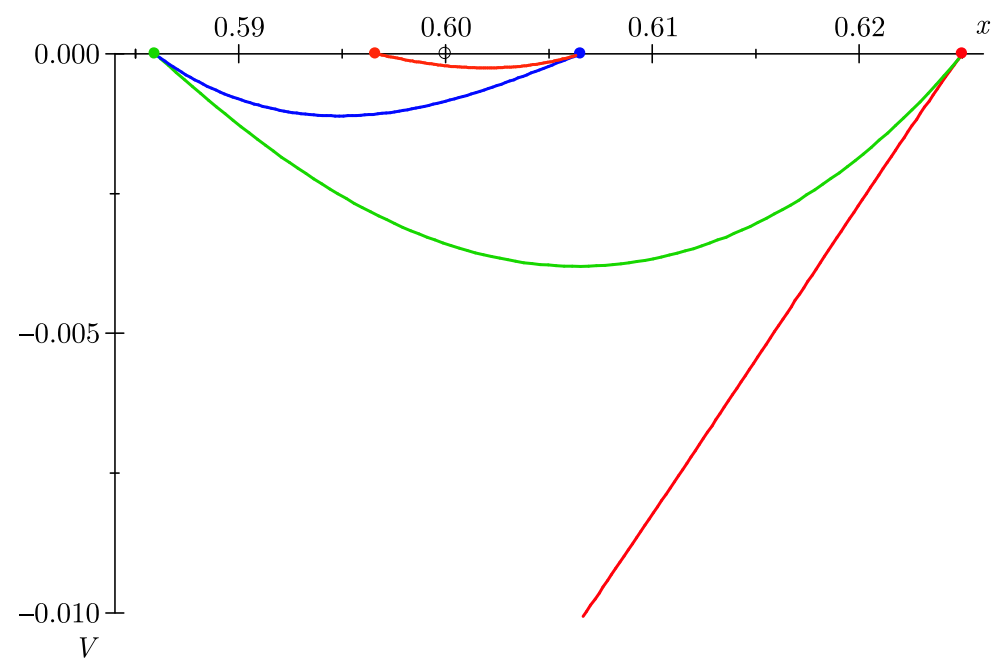

Figure 10. Logistic map potentials for $s=5 / 2$, near the fixed point $x_{*}=3 / 5$.

Rather than plot the potentials, another useful way to envision the dynamics is to construct a phase-space trajectory [5]. This is easily done for those cases where the trajectories are known in closed-form. For cases where numerical analysis is required, it is also relatively straightforward to construct graphs of the phase-space curves using the series solutions, augmented by continuation through use of the functional equations, although for the phase space the relevant functional equation is that for the momentum (note that $p=2 v$ for the mass units we have chosen). This is the square-root of the potential equation (2.3), namely,

$$
p\left(f_{1}(x, s), s\right)=\left(\frac{d}{d x} f_{1}(x, s)\right) p(x, s) .
$$

(In the mathematical literature, an equation of this form is known as a "Julia functional equation" $[12,13]$.) The resulting momentum is multi-valued, in general, with the various branches given by analogues of the recursion relation (4.5), appropriately signed. That is to say,

$$
p_{\underbrace{ \pm \pm \cdots \pm}_{n \text { times }}}(x)= \pm \sqrt{s(s-4 x)} p_{\underbrace{ \pm \pm \cdots \pm}_{n-1 \text { times }}}\left(x_{ \pm}\right),
$$


with $x_{ \pm}$as defined in (4.4). Some of these are actually complex-valued, so for real trajectories, it is necessary to choose from among the $2^{n}$ possibilities at each stage of the recursion. (See the discussion in [3], especially for the $s=10 / 3$ case.) For $2<s \leq 3$, and for $s=4$, the complete infinite sequence of real-valued momentum branches is given recursively by

$$
p_{n}(x)=-\sqrt{s(s-4 x)} p_{n-1}\left(\frac{1}{2}+\frac{1}{2} \sqrt{1-4 x / s}\right) .
$$

For $3<s<4$, it is necessary to consider additional branches from among the $p_{ \pm \pm \cdots \pm}(x)$.

I illustrate this by plotting the first fifteen branches of a zero-energy phase-space trajectory for the marginal $s=3$ case. In some ways this is representative of all $s>2$ cases. Among other things, the plot shows that the system is quasi-Hamiltonian [5] since the resulting trajectory is not single-valued. On the other hand, the trajectory possesses such elegant features that I cannot help but make the suggestion that the underlying analytic potential is somehow fundamental, perhaps even expressible as a known function.

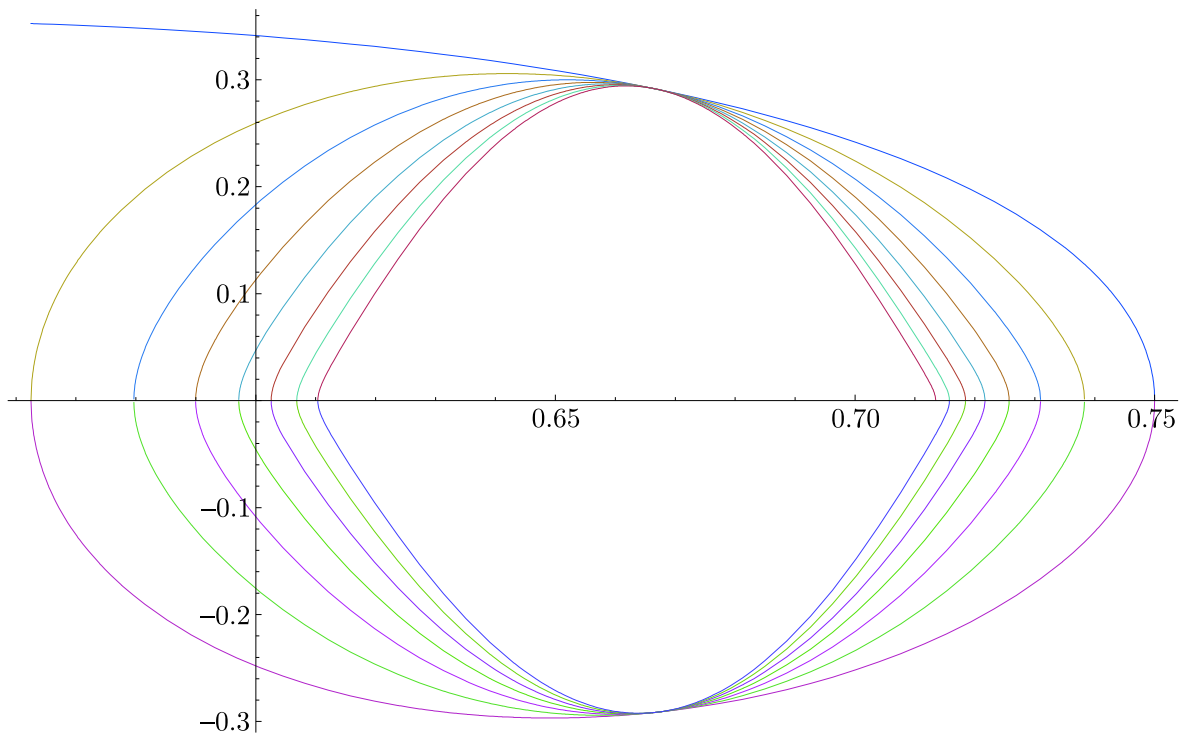

Figure 11. Phase-space trajectory for $s=3$, as given by $d x / d t$ versus $x$. As $t$ advances, the trajectory converges horizontally, but not vertically, towards $x_{*}=\frac{2}{3}$.

One feature that stands out in the graph is the value of $p_{n}\left(x_{*}\right)$ for $x_{*}=2 / 3$. From the recursion relation in this $s=3$ case,

$$
p_{n}(x)=-\sqrt{3(3-4 x)} p_{n-1}\left(\frac{1}{2}+\frac{1}{2} \sqrt{1-4 x / 3}\right),
$$

it follows that $p_{n}(2 / 3)=-p_{n-1}(2 / 3)$, exactly. Thus, while the phase-space trajectory contracts horizontally, towards the asymptotic fixed point at $x_{*}=2 / 3$, the vertical height at $x_{*}=2 / 3$ is fixed and nonzero. Numerically, we find $|d x / d t|_{x=2 / 3}=0.291464$. Remarkably, the transit times for the corresponding $V_{n \geq 1}$ branches of the potential are all $\Delta t=1$, even though those branches also shrink to zero width as $n \rightarrow \infty$, but with fixed depth, $V_{n}(2 / 3)=-0.0849511$.

For additional details, and numerical results, please see $[4,5,3]$. However, I do not wish to give the impression that there is nothing further to be done here. To determine the large time behavior of maps, there is much to be done. While the asymptotic form of $\Psi^{-1}$ can be determined for non-chaotic values of $s$, I do not know what it is for generic, chaotic versions of the logistic map. The one chaotic case where it is known exactly, in simple closed-form (i.e. $s=4$ ), is easily understood, but raises many questions about the behavior for other nearby values of $s$. 


\section{A Series for the $s=1$ logistic map potential}

For this case the appropriate limit of (4.2) gives

$$
\begin{aligned}
V(x, 1) \equiv & \lim _{s \rightarrow 1} V(x, s)=-x^{4}-2 x^{5}-4 x^{6}-\frac{25}{3} x^{7}-\frac{215}{12} x^{8}-\frac{589}{15} x^{9}-\frac{7813}{90} x^{10} \\
& -\frac{60481}{315} x^{11}-\frac{11821}{28} x^{12}+O\left(x^{13}\right) .
\end{aligned}
$$

More systematically, let

$$
V(x, 1)=-x^{4}\left(1+\sum_{n=1}^{\infty} c_{n} x^{n}\right), \quad c_{1}=2, \quad c_{2}=4, \quad c_{3}=\frac{25}{3}, \quad \ldots,
$$

and solve by iteration the functional equation that should be obeyed by $V(x, 1)$, namely,

$$
V(x(1-x), 1)=(1-2 x)^{2} V(x, 1) .
$$

If the formal series (A.1) is constructed to $O\left(x^{25}\right)$ or so, it appears to have a radius of convergence of $R \approx 1 / 2$. But, this convergence is seen to be illusory to higher orders. Different behavior sets in around $O\left(x^{30}\right)$, where the successive $\left|c_{n}\right|^{1 / n}$ used in the limsup determination of $R,(4.3)$, begin to grow linearly, on average, for $n>30$, as shown here (purple curve, with wiggles).



Figure 12. $\left|c_{n}\right|^{1 / n}$ for $s=1$ and $1 \leq n \leq 500$. The blue horizontal is 2 , while the sienna line is $\left(2^{-n / 2} e^{-3 n / 2} n !\right)^{1 / n}$.

That is to say, $\left|c_{n}\right| \sim L^{n} \exp (n \ln n)$ for large $n$, with $L \approx \frac{16}{270} \approx \frac{1}{\sqrt{2} e^{5 / 2}}=5.80429 \times 10^{-2}$. This immediately brings to mind Stirling's formula, $n ! \sim \sqrt{2 \pi n}\left(\frac{n}{e}\right)^{n}$, and in fact, a comparison of $c_{n}$ with $f_{n} \equiv 2^{-n / 2} e^{-3 n / 2} n$ ! is striking, as shown in Fig. 12. (The most important feature in the figure is agreement between the averaged slopes. A better overall fit to the $c_{n}$, on average, is achieved by shifting the sienna line slightly to the left, for example by including an additional $\sqrt{n}$ factor in $f_{n}$.)

All this is compelling evidence that (A.1) itself is not convergent, but rather an asymptotic series. This is consistent with general results in the mathematical literature [8, 13]. Indeed, 
the $f_{n}$ "fit" to $c_{n}$ can be obtained from an asymptotic approximation of a simple integral:

$$
\begin{aligned}
\mathcal{I}(x) & \equiv \int_{0}^{\infty} e^{-y} \frac{1}{1-\frac{x y}{\sqrt{2 e^{3}}}} d y=-\frac{\sqrt{2}}{x} \exp \left(\frac{3 x-2 \sqrt{2} e^{\frac{3}{2}}}{2 x}\right) \operatorname{Ei}\left(1,-\frac{1}{x} \sqrt{2} e^{\frac{3}{2}}\right) \\
& =\int_{0}^{\infty} e^{-y} \sum_{n=0}^{\infty} x^{n} 2^{-n / 2} e^{-3 n / 2} y^{n} d y \sim \sum_{n=0}^{\infty} f_{n} x^{n} .
\end{aligned}
$$

Taking the Cauchy principal value for $\mathcal{I}(x)$ gives finite numerical results for all $x$. These results might be useful to compute corrections to the polynomial approximation for $V(x, 1)$ as constructed from truncating the series (A.1). I leave further analysis of this interesting but peculiar case to the reader.

\section{Acknowledgements}

I would like to thank the organizers of the workshop on Supersymmetric Quantum Mechanics and Spectral Design, Centro de Ciencias de Benasque Pedro Pascual, for the excellent job they did, and for giving me the opportunity to talk about this work. I also thank Andrzej Veitia and Cosmas Zachos for sharing their thoughts about functional evolution methods, and the anonymous referees for suggestions and questions that led to improvements in the manuscript. Finally, I thank the CERN Theoretical Physics Group for its gracious hospitality and generous support during my sabbatical in 2010. This work was also supported in part by NSF Award 0855386.

\section{References}

[1] Beverton R.J.H., Holt S.J., On the dynamics of exploited fish populations, Fishery Investigations Series II, Vol. XIX, Ministry of Agriculture, Fisheries and Food, 1957.

[2] Collet P., Eckmann J.-P., Iterated maps on the interval as dynamical systems, Progress in Physics, Vol. 1, Birkhäuser, Boston, Mass., 1980.

[3] Curtright T., Veitia A., Logistic map potentials, Phys. Lett. A 375 (2011), 276-282, arXiv:1005.5030.

[4] Curtright T., Zachos C., Evolution profiles and functional equations, J. Phys. A: Math. Theor. 42 (2009), 485208, 16 pages, arXiv:0909.2424.

[5] Curtright T., Zachos C., Chaotic maps, hamiltonian flows and holographic methods, J. Phys. A: Math. Theor. 43 (2010), 445101, 15 pages, arXiv:1002.0104.

[6] Curtright T., Zachos C., Renormalization group functional equations, Phys. Rev. D 83 (2011), 065019, 17 pages, arXiv:1010.5174.

[7] Devaney R.L., An introduction to chaotic dynamical systems, 2nd ed., Addison-Wesley Studies in Nonlinearity, Addison-Wesley Publishing Company, Advanced Book Program, Redwood City, CA, 1989.

[8] Erdös P., Jabotinsky E., On analytic iteration, J. Analyse Math. 8 (1960), 361-376.

[9] Feigenbaum M.J., Quantitative universality for a class of nonlinear transformations, J. Statist. Phys. 19 (1978), 25-52.

[10] Geritz S.A.H., Kisdi E., On the mechanistic underpinning of discrete-time population models with complex dynamics, J. Theor. Biology 228 (2004), 261-269.

[11] Goldstone J., Field theories with "superconductor" solutions, Nuovo Cimento 19 (1961), 154-164.

[12] Julia G., Mémoire sur l'itération des fonctions rationnelles, Journ. de Math. (8) 1 (1918), 47-245.

[13] Kuczma M., Choczewski B., Ger R., Iterative functional equations, Encyclopedia of Mathematics and its Applications, Vol. 32, Cambridge University Press, Cambridge, 1990.

[14] Nambu Y., Quasi-particles and gauge invariance in the theory of superconductivity, Phys. Rev. 117 (1960), 648-663.

[15] Patrascioiu A., Classical Euclidean solutions, Phys. Rev. D 15 (1977), 3051-3053.

[16] Poincaré H., Sur une classe étendue de transcendantes uniformes, C.R. Acad. Sci. Paris 103 (1886), 862-864.

[17] Schröder E., Über iterierte Funktionen, Math. Ann. 3 (1870), 296-322.

[18] Skellam J.G., Random dispersal in theoretical populations, Biometrika 38 (1951), 196-218. 